\title{
Postoperative Serum CEA Level is a More Significant Prognostic Factor than Post/Preoperative Serum CEA Ratio in Non-small Cell Cancer Patients
}

\author{
Masaki Tomita, Takanori Ayabe, Eiichi Chosa, Kunihide Nakamura*
}

\begin{abstract}
Background: In non-small cell lung cancer (NSCLC) patients with preoperative high serum carcinoembryonic antigen (CEA) level, patients with a persistently high serum CEA level after surgery have been reported to have a poor prognosis. In addition, in other cancers, the post/preoperative serum CEA ratio has been reported as a useful parameter. Materials and Methods: We enrolled 123 NSCLC patients with preoperative high CEA levels $(\geq 5 \mathrm{ng} / \mathrm{mL}$ ) who underwent curative surgery between 2004 and 2011. Prognostic significance of postoperative serum CEA level and the CEA ratio was examined. Results: The 5-year survival of patients with persistently high serum CEA level after surgery was poor. On the other hand, patients with normal postoperative serum CEA levels had significant favorable prognosis. The patients with CEA ratio $>1$ had poor prognosis, however the number was only $7(5.7 \%)$. The 5 -year survival rates of patients with other subgroup based on the CEA ratio $(0.5 \geq$ CEA ratio and $0.5 \leq$ CEA ratio $\leq 1)$ was similar. Multivariate analysis revealed prognostic significance for the postoperative serum CEA level but not the CEA ratio. Conclusions: For NSCLC patients with preoperative high serum CEA level, their postoperative serum CEA levels is a more significant prognostic factor than the post/preoperative serum CEA ratio.
\end{abstract}

Keywords: non-small cell lung cancer - serum CEA level - preoperative - postoperative - CEA ratio - prognosis

Asian Pac J Cancer Prev, 16 (17), 7809-7812

\section{Introduction}

Carcinoembryonic antigen (CEA) is the most widely used tumor marker in patients with non-small cell lung cancer (NSCLC) (Grunnet et al., 2012). Several studies have suggested that preoperative serum CEA is an independent prognostic factor for NSCLC (Sawabata et al., 2002; Okada et al., 2004; Tomita et al., 2004; Grunnet et al., 2012). Okada et al. analyzed 1000 patients with clinical stage I NSCLC and found that the preoperative serum CEA level was an independent prognostic factor (Okada et al., 2004). Sawabata et al. also reached the same conclusion after studying 273 patients with clinical stage I disease (Sawabata et al., 2002). Our previous study also showed the same results (Tomita et al., 2004).

Moreover, a number of studies also report that postoperative CEA levels is associated with the oncologic outcomes of NSCLC (Okada et al., 2004, Sawabata et al., 2002, 2004; Nonaka et al., 2004; Tomita et al., 2005).

In addition to postoperative serum CEA level, previous studies also reported the usefulness of the post/preoperative serum CEA ratio which was defined as the postoperative serum CEA value divided by the preoperative serum CEA value in other cancers (Hotta et al., 2006; Hotta et al., 2014). To our knowledge, there are no studies about the prognostic significance of the post/ preoperative serum CEA ratio in NSCLC. Therefore, in the present study, we retrospectively investigated the prognostic significance of the post/preoperative serum CEA ratio using NSCLC patients with preoperative high serum CEA level. Furthermore, we compared the usefulness between postoperative serum CEA level and post/preoperative serum CEA ratio.

\section{Materials and Methods}

This retrospective study had institutional review board approval, and the need to obtain patient consent was waived. Consecutive NSCLC patients with preoperative high serum CEA level who underwent surgery from 2004 to 2011 in our hospital were enrolled into the present retrospective study. The following patients were excluded: (1) patients who had not received complete resection which consisted of either a lobectomy or a pneumonectomy together with the regional lymph nodes dissection, (2) patients who died of other diseases after surgery, and (3) patients who lost to follow-up. One hundred and twenty-three consecutive NSCLC patients 
Masaki Tomita et al

with preoperative high serum CEA level were enrolled into the present retrospective study. The clinicopathologcal factors of patients were shown in Table 1. The preoperative serum CEA level was measured using the two-site immunoenzymometric assay; the normal upper limit for this assay was $5.0 \mathrm{ng} / \mathrm{mL}$. All patients had preoperative high serum CEA level. The time interval between preoperative serum CEA examination and surgical resection was less than 2 weeks in all the patients. The postoperative serum CEA level was also measured during 1 to 2 months after surgery for each patient. The CEA ratio which was defined as the postoperative serum CEA value divided by the preoperative serum CEA value was measured in all patients.

Pathological (p) tumor-node-metastasis (TNM) staging was recorded in all patients based on the 7th edition of the American Joint Committee on Cancer (AJCC)/ Union for International Cancer Control (UICC) classification. Follow-up information, including cause of death, was ascertained through a review of clinic notes and direct or family contact. The disease-specific survival curves of the patients were plotted by using the Kaplan-Meier method and analyzed using the log-rank test. The Cox regression hazard model was used for univariate and multivariate analyses to assess the prognostic value of postoperative serum CEA level and CEA ratio. Statistical calculations were conducted with JMP (SAS Institute Inc., Cary, NC, USA) and values of $p$ less than 0.05 were accepted as being significant.

\section{Results}

The postoperative disease-specific 5-year survival rates based on postoperative serum CEA level in the patients with postoperative serum CEA level $<5.0(\mathrm{n}=95)$ and $\geq 5.0(n=39)$ were $67.3 \%$ and $24.9 \%$, respectively (Figure 1). This difference was significant $(\mathrm{p}<0.0001)$.

According to previous study $(8,9)$, the cutoff level of CEA ratio was set at 1 . However, in our series, there are only $7 / 123$ patients $(5.7 \%)$ with CEA ratio $>1$. Therefore, based on the CEA ratio, patients were subdivided into 3 groups; group $A(n=72)$ : CEA ratio $<0.5$, group $B(n=44)$ : $0.5 \leq$ CEA ratio $\leq 1$ and group $C(n=7)$ : CEA ratio $>1$. As shown in $v 2$, the disease-specific 5-year survival of group A, B and C were $60.1 \%, 51.7 \%$, and $0 \%$, respectively.

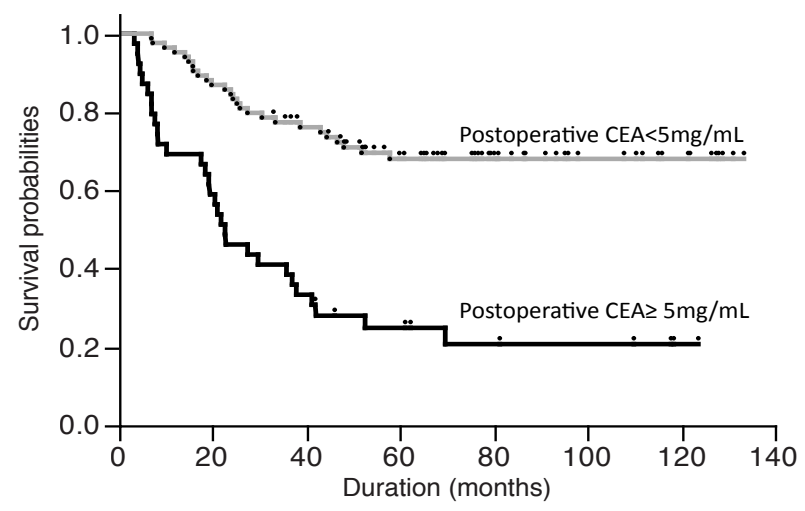

Figure 1. Survival of Patients Based on Postoperative Serum CEA Level

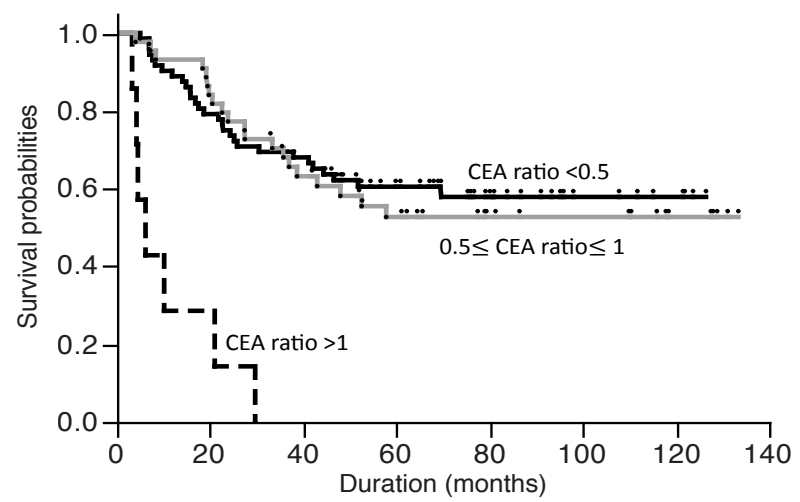

Figure 2. Survival of Patients Based on Post/ preoperative Serum CEA Ratio

Table 1. Comparison of Clinical Characteristics

\begin{tabular}{lcc}
\hline Variables & & Number of patients \\
\hline Age & $<65$ & 32 \\
& $\geq 65$ & 91 \\
Gender & Male & 84 \\
& Female & 39 \\
Histology & Adenocarcinoma & 90 \\
& others & 33 \\
pStage & I & 75 \\
& II-III & 48 \\
pT status & pT1 & 65 \\
& pT2-3 & 58 \\
pN status & pN0 & 89 \\
& pN1-2 & 34 \\
Smoking & ever & 92 \\
& never & 31 \\
CEA & $<10$ & 79 \\
& $\geq 10$ & 44
\end{tabular}

CEA: carcinoembryonic antigen

Table 2. Univariate analysis

\begin{tabular}{llccc}
\hline Variables & \multicolumn{5}{c}{ Risk ratio } & $95 \%$ CI & p Value \\
\hline Age & $\geq 65$ & 1.000 & & \\
\multirow{2}{*}{ Gender } & $<65$ & 0.776 & $0.401-1.402$ & 0.413 \\
& Male & 1.000 & & \\
Histology & Female & 0.392 & $0.187-0.744$ & 0.003 \\
& Adeno & 1.000 & & \\
Smoking & others & 1.910 & $1.085-3.268$ & 0.026 \\
& never & 1.000 & & \\
pT status & ever & 3.557 & $1.650-9.277$ & 0.001 \\
& pT2-3 & 1.000 & & \\
pN status & pN0 & 1.558 & $0.922-2.653$ & 0.098 \\
& pN1-2 & 2.579 & $0.880-2.727$ & 0.022 \\
pre CEA & $<10$ & 1.000 & & \\
& $\geq 10$ & 1.695 & $0.993-2.867$ & 0.053 \\
CEA ratio & $<0.5$ & 1.000 & & \\
& $0.5-1$ & 1.106 & $0.617-1.943$ & 0.731 \\
& $\geq 1$ & 9.910 & $3.877-22.318$ & $<0.0001$ \\
post CEA & $<5$ & 1.000 & & \\
& $\geq 5$ & 4.023 & $2.367-6.881$ & $<0.0001$ \\
\hline
\end{tabular}

CI: Confidence interval, CEA: carcinoembryonic antigen

Although the survival was not different between group $\mathrm{A}$ and $\mathrm{B}$, the survival of group $\mathrm{C}$ were significantly unfavorable $(\mathrm{p}<0.0001)$.

The result of univariate analysis is summarized in 
Table 3. Multivariate Analysis

\begin{tabular}{|c|c|c|c|c|}
\hline Variables & & Risk ratio & $95 \% \mathrm{CI}$ & $\mathrm{p}$ Value \\
\hline \multirow[t]{2}{*}{$\overline{\text { Age }}$} & $\geq 65$ & 1.000 & & \\
\hline & $<65$ & 0.550 & $0.267-1.071$ & 0.080 \\
\hline \multirow[t]{2}{*}{ Gender } & Male & 1.000 & & \\
\hline & Female & 0.656 & $0.244-1.540$ & 0.350 \\
\hline \multirow[t]{2}{*}{ Histology } & Adeno & 1.000 & & \\
\hline & others & 1.048 & $0.530-2.026$ & 0.890 \\
\hline \multirow[t]{2}{*}{ Smoking } & never & 1.000 & & \\
\hline & ever & 1.869 & $0.602-6.219$ & 0.283 \\
\hline \multirow[t]{2}{*}{ pT status } & pT1 & 1.000 & & \\
\hline & pT2-3 & 1.011 & $0.549-1.873$ & 0.971 \\
\hline \multirow[t]{2}{*}{$\mathrm{pN}$ status } & $\mathrm{pNO}$ & 1.000 & & \\
\hline & $\mathrm{pN} 1-2$ & 2.406 & $1.223-4.581$ & 0.012 \\
\hline \multirow[t]{2}{*}{ pre CEA } & $<10$ & 1.000 & & \\
\hline & $\geq 10$ & 1.155 & $0.582-2.253$ & 0.676 \\
\hline \multirow[t]{3}{*}{ CEA ratio } & $<0.5$ & 1.000 & & \\
\hline & $0.5-1$ & 0.767 & $0.349-1.635$ & 0.493 \\
\hline & $\geq 1$ & 3.051 & $0.872-9.879$ & 0.079 \\
\hline \multirow[t]{2}{*}{ post CEA } & $<5$ & 1.000 & & \\
\hline & $\geq 5$ & 3.242 & $1.625-6.547$ & 0.001 \\
\hline
\end{tabular}

CI: Confidence interval, CEA: carcinoembryonic antigen

Table 2. The gender, histology (adenocarcinoma vs. others), smoking status (ever vs. never), $\mathrm{pN}$ status (pN0 vs. pN1-2) and postoperative serum CEA level (normal vs. high) were related to patient prognosis. Although CEA ratio $>1$ had a $p$ value of $<0.0001$ when compared with CEA ratio $<0.5,0.5 \leq$ CEA ratio $\leq 1$ had a $p$ value of 0.675 for the same comparison. The result of multivariate analysis is also summarized in Table 3 . The pN status and postoperative serum CEA level were independent prognostic determinants, but not others, including the CEA ratio.

\section{Discussion}

Serum CEA level is a widely studied and easy to assess during the preoperative or postoperative periods. In the present study, we included NSCLC patients with preoperative high serum CEA level. Patients with high CEA level are more common in men, ever smokers and adenocarcinoma in our series. Previous studies showed that patients with high serum CEA level tend to have advanced disease (Takamochi et al., 2000, Yamazaki et al., 2007). However, our series is not always more common in advanced diseases.

Serum CEA level can clearly and consistently be judged as normal or abnormal if a cutoff value is set. Several studies revealed the prognostic significance of preoperative serum CEA level (Sawabata et al., 2002; Okada et al., 2004; Tomita et al., 2004; Grunnet et al., 2012). Okada et al. reported their experience with 1000 consecutive resections for stage I NSCLC and concluded that perioperative measurement of serum CEA concentrations yields information valuable for detecting patients with high risk of poor survival (Okada et al., 2004). Sawabata et al. assessed 297 patients with clinical stage I NSCLC and found that serum CEA level is a useful predictor of survival of patients with stage I NSCLC (Sawabata et al., 2002). Our previous study also found that patients with preoperative high serum CEA level had the poor survival (Tomita et al., 2004).

With regard to postoperative serum CEA level, Okada et al. reported that $368 / 1000$ patients $(36.8 \%)$ had preoperative high serum CEA levels, and normalization of CEA levels after surgery is a favorable prognostic factor in these patients (Okada et al., 2004). Sawabata et al. also found that a persistently high serum CEA level after surgery is an indicator of a very poor prognosis (Sawabata et al., 2002; Sawabata et al., 2004). Our previous study also revealed that normalization of serum CEA level after surgery are significant prognostic determinants in 82 NSCLC patients with preoperative high serum CEA levels (Tomita et al., 2005) and the present study also revealed a similar result. Whether a persistently high serum CEA indicates inadequate resection, under staging, or more aggressive tumor biology is undetermined. It is easy to consider that persistently high CEA levels after surgery may be related to occult residual disease. Previous studies (Okada et al., 2004; Tomita et al., 2005) also discussed as follows: If the surgical extirpation of a tumor was complete and if no residual tumor was present, there was no source of CEA production in the body and a regression of CEA followed its metabolic clearance rate. If residual tumor cells exist, the serum CEA level does not normalize, and the serum level is maintained at a plateau that represents the number of residual tumor cells. Therefore, postoperative high serum CEA level might mean existing residual tumor cells in the body. In this view, many of NSCLC patients with preoperative high and postoperative normal serum CEA level might have local disease which is cured by surgical extirpation, whereas those with postoperative high serum CEA level might have unrecognized extrapulmonary disease.

Regarding the post/preoperative serum CEA ratio, it has also been reported that the CEA ratio is a factor associated with a poor prognosis for survival after surgery in colorectal cancer patients with synchronous unresectable liver metastases (Hotta et al., 2006). Same authors also clarified the usefulness of the post/preoperative serum CEA ratio as a predictor of the prognosis after surgery for stage III rectal cancer patients. (Hotta et al., 2014) They also showed that a post/preoperative serum CEA ratio $>1$ may be more effective for predicting liver metastasis than other types of metastasis (Hotta et al., 2014). It has also been reported that the reduction ratio of pre- to post-chemoradiotherapy (CRT) serum CEA levels may be a prognostic factor for disease-free survival in rectal cancer patients with a pre- CRT CEA of more than $6 \mathrm{ng} / \mathrm{ml}$ (Kim et al., 2011). To our knowledge, there are no studies about the prognostic significance of CEA ratio in NSCLC. In our result, NSCLC patients with CEA ratio $>1$ had significant poor prognosis. However, CEA ratio $>1$ group population was small and accounted for only $5.7 \%$ of patients. Moreover, the survival among patients with other group was similar. In other word, CEA ratio is useful only for $5.7 \%$ of NSCLC patients with preoperative high serum CEA level. In addition, our result of multivariate analysis showed the prognostic significance of postoperative serum CEA level but not CEA ratio. Therefore, from our results, postoperative serum CEA level is more useful than CEA ratio for 


\section{Masaki Tomita et al}

NSCLC patients with preoperative high serum CEA level. Hotta et al. reported that CEA ratio is a predictor of the prognosis after surgery for stage III rectal cancer patients, and the number of their patients with CEA ratio $>1$ was 18/114 (15.8\%) (Hotta et al., 2014). The reason for the difference in the frequency of patients with CEA ratio $>1$ is unknown, but might be due to differences in cancer organ and stage of patients' population at least in part. It is necessary to pay attention to the limitation of this study by the small number of patients, and our current findings should be confirmed in larger studies.

Previous authors considered that patients with persistently high serum CEA level after surgery are good candidates for adjuvant chemotherapy (Sawabata et al., 2002; Okada et al., 2004; Sawabata et al., 2004; Nonaka et al, 2004; Tomita et al., 2005). However, there are no studies whether adjuvant chemotherapy is effective for these patients or not. Further studies in this area are warranted.

In conclusion, We failed to find the prognostic significance of post/preoperative serum CEA ratio for NSCLC patients with preoperative high serum CEA level. The postoperative serum CEA level is an extremely prognostic factor than post/preoperative serum CEA ratio for these NSCLC patients.

\section{References}

Grunnet M, Sorensen JB (2012). Carcinoembryonic antigen (CEA) as tumor marker in lung cancer. Lung Cancer, 76, 138-43.

Hotta T, Takifuji K, Uchiyama K, et al (2006). Potential predictors of survival after surgery for colorectal cancer patients with synchronous unresectable liver metastases. Oncology Reports, 16, 1369-74.

Hotta T, Takifuji K, Yokoyama S, et al (2014). Impact of the post/preoperative serum CEA ratio on the survival of patients with rectal cancer. Surgery Today, 44, 2106-15.

Kim CW, Yu CS, Yang SS, et al (2011). Clinical significance of pre- to post-chemoradiotherapy s-CEA reduction ratio in rectal cancer patients treated with preoperative chemoradiotherapy and curative resection. Annals of Surgical Oncology, 18, 3271-7.

Nonaka M, Kataoka D, Yamamoto S, et al (2004). Pre- and postoperative serum carcinoembryonic antigen in primary lung adenocarcinoma. Annals of Thoracis and Cardiovascular Surgery, 10, 281-4.

Okada M, Nishio W, Sakamoto T, et al (2004). Prognostic significance of perioperative serum carcinoembryonic antigen in non-small cell lung cancer: analysis of 1,000 consecutive resections for clinical stage I disease. The Annals of Thoracic Surgery, 78, 216-21.

Sawabata N, Ohta M, Takeda S, et al (2002). Serum carcinoembryonic antigen level in surgically resected clinical stage I patients with non-small cell lung cancer. The Annals of Thoracic Surgery, 74, 174-9.

Sawabata N, Maeda H, Yokota S, et al (2004). Postoperative serum carcinoembryonic antigen levels in patients with pathologic stage IA nonsmall cell lung carcinoma: subnormal levels as an indicator of favorable prognosis. Cancer, 101, 803-9.

Takamochi K, Nagai K, Suzuki K, et al (2000). Clinical predictors of $\mathrm{N} 2$ disease in non-small cell lung cancer. Chest, 117, 1577-82.
Tomita M, Matsuzaki Y, Edagawa M, et al (2004). Prognostic significance of preoperative serum carcinoembryonic antigen level in lung adenocarcinoma but not squamous cell carcinoma. Annals of Thoracis and Cardiovascular Surgery, 10, 76-80.

Tomita M, Matsuzaki Y, Shimizu T, et al (2005). Prognostic determinants for lung cancer patients with preoperative high serum carcinoembryonic antigen levels. Thoracic and Cardiovascular Surgeon, 53, 300-4.

Wang CY, Huang MS, Huang MH, et al (2010). Persistently high serum carcinoembryonic antigen levels after surgery indicate poor prognosis in patients with stage I non-small-cell lung cancer. Journal of Surgical Research, 163, 45-50.

Yamazaki K, Yoshino I, Yohena T, et al (2007). Clinically predictive factors of pathologic upstaging in patients with peripherally located clinical stage IA non-small cell lung cancer. Lung Cancer, 55, 365-9. 